\title{
Safety of an Alkalinizing Buffer Designed for Inhaled Medications in Humans
}

\author{
Michael D Davis RRT, Brian K Walsh MBA RRT-NPS RPFT FAARC, \\ Scott T Dwyer, Casey Combs, Nico Vehse MD, Alix Paget-Brown MD, \\ Thomas Pajewski MD PhD, and John F Hunt MD
}

\begin{abstract}
BACKGROUND: Airway acidification plays a role in disorders of the pulmonary tract. We hypothesized that the inhalation of alkalinized glycine buffer would measurably alkalinize the airways without compromising lung function or causing adverse events. We evaluated the safety of an inhaled alkaline glycine buffer in both healthy subjects and in subjects with stable obstructive airway disease. METHODS: This work includes 2 open-label safety studies. The healthy controls were part of a phase 1 safety study of multiple inhalations of low-dose alkaline glycine buffer; nebulized saline was used as a comparator in 8 of the healthy controls. Subsequently, a phase 2 study in subjects with stable obstructive airway disease was completed using a single nebulized higher-dose strategy of the alkaline inhalation. We studied 20 non-smoking adults (10 healthy controls and 10 subjects with obstructive airway disease), both at baseline and after inhalation of alkaline buffer. We used spirometry and vital signs as markers of clinical safety. We used changes in fraction of exhaled nitric oxide (NO) and exhaled breath condensate (EBC) pH as surrogate markers of airway pH modification. RESULTS: Alkaline glycine inhalation was tolerated by all subjects in both studies, with no adverse effects on spirometric parameters or vital signs. Airway alkalinization was confirmed by a median increase in $\mathrm{EBC} \mathrm{pH}$ of $0.235 \mathrm{pH}$ units (IQR 0.56-0.03, $P=.03)$ in subjects after inhalation of the higher-dose alkaline buffer $(2.5 \mathrm{~mL}$ of $120 \mathrm{mmol} / \mathrm{L}$ glycine). CONCLUSIONS: Alkalinization of airway lining fluid is accomplished with inhalation of alkaline glycine buffer and causes no adverse effects on pulmonary function or vital signs. Key words: airway acidification; glycine; buffer; COPD; exhaled nitric oxide; NO; exhaled breath condensate; airway pH. [Respir Care 2013;58(7):1226-1232. (C) 2013 Daedalus Enterprises]
\end{abstract}

\section{Introduction}

The regulation of airway $\mathrm{pH}$ plays a role in the pathogenesis of obstructive lung diseases. Airway acidification,

Mr Davis is affiliated with the Adult Health and Nursing System, Virginia Commonwealth University, Richmond, Virginia. Mr Dwyer, Mrs Combs, Dr Vehse, and Dr Hunt are affiliated with the Division of Pediatric Respiratory Medicine; Dr Paget-Brown is affiliated with the Division of Neonatology; and Dr Pajewski is affiliated with the Department of Anesthesiology, University of Virginia, Charlottesville, Virginia. At the time of this study Mr Walsh was affiliated with the Children's Medical Center of Dallas, Dallas, Texas; he is now with Boston Children's Hospital, Boston, Massachusetts.

Dr Hunt has disclosed a relationship with Respiratory Research Inc, Austin, Texas, which manufactures exhaled breath condensate collection equipment. Dr Hunt and the University of Virginia have intellectual property interest in airway $\mathrm{pH}$ diagnosis and therapy. Mr Davis was caused by both intrinsic and extrinsic factors, is associated with neutrophilic and eosinophilic inflammation, bronchospasm, bronchial hyper-reactivity, ciliary dysfunction, ep-

partly supported by National Institute of Nursing Research grant K99/ R00 NR012016. This research was funded in part by the National Institute of Health and the University of Virginia Philip Morris Tobacco Research Fund.

Mr Davis presented a version of this paper at the 57th AARC Congress, held November 5-8, 2011, in Tampa, Florida.

Correspondence: Michael D Davis RRT, Adult Health and Nursing System, Medical College of Virginia, School of Nursing Building, 4th Floor, Room 4010c, 1100 East Leigh Street, PO Box 980567, Richmond VA 23298-0567. E-mail: mdavis35@vcu.edu.

DOI: $10.4187 /$ respcare. 01753 


\section{Safety of an Alkalinizing Buffer Designed for Inhaled Medications in Humans}

ithelial dysfunction, augmented oxidative damage, abnormal fluid transport, inhibition of transport of cationic drugs such as albuterol, and alteration of cellular death pathways, including inhibition of apoptosis. ${ }^{1}$ Knowledge of the role of airway $\mathrm{pH}$ in pulmonary health, along with the development of devices and techniques to measure it, has created interest in treatment of airway $\mathrm{pH}$ disturbances. Improved ability to treat or to potentially reverse acidic airway pathology by means of therapeutic alteration of airway $\mathrm{pH}$ could have an impact in respiratory medicine. The ability to normalize airway $\mathrm{pH}$ via inhalation ${ }^{2}$ may allow introduction of new pulmonary therapeutics.

Airway lining fluid (ALF) acidity can be qualitatively determined noninvasively via the collection of exhaled breath condensate (EBC) and the measurement of its $\mathrm{pH} .{ }^{3}$ Assays for the measurement of EBC $\mathrm{pH}$ have been developed for patients of all ages and sizes, including those receiving mechanical ventilation. ${ }^{4-7} \mathrm{EBC} \mathrm{pH}$ normally lies within a mildly alkaline range of 7.5-8.2.7-19 $\mathrm{EBC}$ has a minimal buffer capacity, which allows EBC to assess the presence of volatile acids in ALF, as indicated by a change in its $\mathrm{pH}^{20,21}$ Although a normal EBC $\mathrm{pH}$ does not exclude airway acidity at some level, a low EBC $\mathrm{pH}$ value is highly specific for acidity somewhere within the airway. Using EBC methods, several studies have shown that patients with COPD, ${ }^{13}$ asthma,,${ }^{7,9}$ bronchiectasis, ${ }^{13}$ cystic fibrosis, ${ }^{9}$ and chronic cough ${ }^{15}$ have airway acidification. We incorporated EBC into this study as a noninvasive safety measure to assess for the possibility of excessive alkalinization from our intervention.

Airway $\mathrm{pH}$ also affects fraction of exhaled nitric oxide (FeNO) levels by simple chemistry. As the pH of ALF decreases, commonly present nitrite becomes protonated to nitrous acid, which decomposes to nitric oxide, ${ }^{22}$ which is then in part exhaled. $\mathrm{F}_{\mathrm{ENO}}$ analyzers may be used qualitatively to longitudinally assess the alkalinizing effects of alkaline inhalation therapy through monitoring decreases in $\mathrm{F}_{\mathrm{ENO}}$ levels. ${ }^{2,23}$ The first aim of the present study was to evaluate the safety of an inhaled aerosol of alkaline glycine buffer in healthy controls; we also investigated the potential of this inhaled, aerosolized buffer to alkalinize the ALF pH in both healthy subjects and those with stable obstructive airway disease.

\section{Methods}

\section{Protocol}

Two open-label safety studies were approved by the University of Virginia institutional review board under an Investigational New Drug Application from the United States Food and Drug Administration. Approval and initiation of the second study by the University of Virginia institutional review board was dependent upon successful completion of the first study and results indicating safety

\section{QUICK LOOK}

\section{Current knowledge}

The regulation of airway $\mathrm{pH}$ plays a role in the pathogenesis of obstructive lung disease. Airway acidification is associated with airway inflammation, bronchospasm, and other pathophysiologic derangements. The impact of airway alkalization as a new therapeutic option is unclear.

\section{What this paper contributes to our knowledge}

The inhalation of isotonic alkaline glycine is safe in normals and in patients with chronic lung disease. Alkalization of the airway markedly decreases exhaled breath $\mathrm{pH}$, but did not alter the exhaled nitric oxide level.

of the intervention. We obtained informed consent from all subjects for both studies. The first study recruited 10 healthy volunteers via direct approach from the study team. The study was performed in the General Clinical Research Center. No reimbursement was provided. The second study recruited 10 subjects with stable obstructive lung disease, via referral from their allergist, and was performed in the Allergy/Asthma/Immunology Clinic. Subjects were reimbursed $\$ 100$ for participation. An independent medical safety monitor reviewed the results for each subject daily during the studies. Both studies were executed between 10:00 AM and 2:00 PM Eastern Standard Time.

For the first study (study 1) we acquired (in the following order) baseline $\mathrm{F}_{\mathrm{ENO}}$ levels, $\mathrm{EBC}$ samples, spirometry $\left(\mathrm{FEV}_{1}, \mathrm{FVC}, \mathrm{FEV}_{1} / \mathrm{FVC}\right.$, and forced expiratory flow during the middle half of the FVC maneuver $\left[\mathrm{FEF}_{25-75 \%}\right]$ ), and vital signs (heart rate, breathing frequency, oxygen saturation, and breath sounds) from the subjects. The order in which these measurements were obtained was kept consistent throughout all points of the study, with the exception of EBC, which was collected only before the first inhalation and immediately after the third inhalation. All of the subjects were within healthy limits for spirometry (baseline $>80 \%$ of predicted for all observed values) and vital signs, and had not ingested anything except water for 8 hours prior to the study. Exclusion criteria included a $>5$ pack-year smoking history, current pregnancy (all female subjects were either abstinent, post-menopausal, or practicing adequate contraception), a history of pulmonary disease (verified through verbal medical history), or acute illness (verified by clinical history or reported by the subject) within 5 days of study.

The study 1 treatment preparation was an isotonic solution of a sodium chloride diluent mixed with glycine to 


\section{Safety of an Alkalinizing Buffer Designed for Inhaled Medications in Humans}

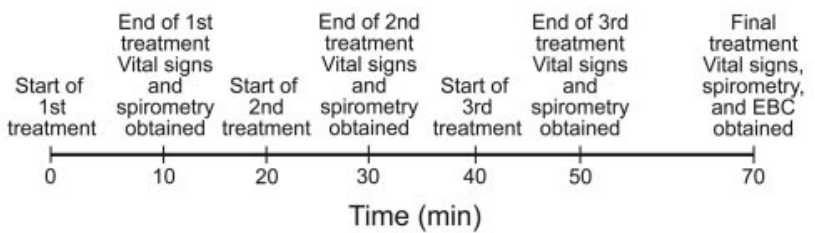

Fig. 1. Timeline of events for study 1.

a concentration of $17.8 \mathrm{mmol} / \mathrm{L}$ at $\mathrm{pH}$ of 10.5 , in $2.5 \mathrm{~mL}$ increments every $20 \mathrm{~min}$, for a total of 3 nebulizations (Fig. 1). Each nebulization lasted for $10 \mathrm{~min}$, with a 10 minute break in between, during which vital signs, $\mathrm{F}_{\mathrm{ENO}}$ and spirometry were obtained. A final set of physiologic measurements was performed $20 \mathrm{~min}$ after the completion of the study.

After determining safety, in healthy volunteers, of serial incremental inhalations of glycine, a second study (study 2) was conducted in stable subjects with known obstructive lung disease, defined as a documented history of asthma or COPD and an $\mathrm{FEV}_{1}<75 \%$ of predicted on the day of the study. Exclusion criteria included cigarette smoking in the past 6 months, any acute illness within 5 days of the study (verified by clinical history or reported by the subject), or an $\mathrm{FEV}_{1}<50 \%$ of predicted on the day of the study. Eight of these subjects had a current diagnosis of asthma, one had a current diagnosis of chronic bronchitis, and one had diagnoses of both asthma and chronic bronchitis. The ages of the enrolled subjects ranged from 24-62 years old, with a mean of 44 years. This study protocol only varied from the study 1 protocol by using a single $2.5 \mathrm{~mL}$ dose of alkaline diluent with $120 \mathrm{mmol} / \mathrm{L}$ of glycine instead of the 3 lower-concentrations nebulizer treatments used in study 1. Vital signs (heart rate, breathing frequency, oxygen saturation, and breath sounds) were obtained prior to, during, and after the nebulization. $\mathrm{F}_{\mathrm{ENO}}, \mathrm{EBC}$, and spirometry were obtained before and after the nebulization. All measurements were obtained in the same order as in study 1 .

\section{Instrumentation}

$\mathrm{F}_{\mathrm{ENO}}$ was measured at an expiratory flow rate of $50 \mathrm{~mL} / \mathrm{s}$ with a device designed to measure $\mathrm{F}_{\mathrm{ENO}}$ (Niox Mino, Aerocrine, Solna, Sweden). Spirometry was measured in triplicate at each collection point with a spirometer (SDI SBG, Queset Medical, Brockton, Massachusetts). EBC was collected orally during tidal breathing for $7 \mathrm{~min}$, at initial temperature of $-20^{\circ} \mathrm{C}$ with a sampling device (RTube, Respiratory Research, Austin, Texas), without nose clips. All samples underwent gas-standardization with research- grade oxygen for $10 \mathrm{~min}$ at $300 \mathrm{~mL} / \mathrm{min}$ prior to $\mathrm{pH}$ measurement.

EBC pH was measured using an electrode (Orion 8220BNWP PerpHecT Ross combination pH Micro Electrode, Thermo Scientific, Waltham, Massachusetts). The probe was calibrated in standard $\mathrm{pH} 4$ and 7 buffers, and then verified in low-ionic-strength buffers of the same $\mathrm{pH}$, and then $\mathrm{pH}$ of samples was measured immediately after gasstandardization.

Nebulizations were administered for 10 min using a nebulizer (Micromist, Hudson RCI/Teleflex Medical, Research Triangle Park, North Carolina), with a mouthpiece. The nebulizer was powered by $7 \mathrm{~L} / \mathrm{min}$ of room air, a rate at which it averages an output mass median aerodynamic diameter of $2.1 \mu \mathrm{m}$, according to manufacturer. The nebulizer cup was percussed periodically during medication delivery, to minimize residual volume. The residual volume was removed at the end of each 10-min inhalation. Oxygen saturation was obtained using a fingertip pulse oximeter (SET, Massimo, Irvine, California).

\section{Study Medication Specifications}

The alkaline glycine buffer was formulated by a compounding pharmacy under sterile conditions. Sodium chloride and sodium hydroxide were added to a solution of double deionized water and alkaline glycine, in order to attain appropriate isotonicity ( $0.9 \%$ weight/volume), osmolarity (338 mOsmoles), and $\mathrm{pH}$ (9.8) to prevent cough upon nebulization. After receipt of each batch of medication from the pharmacy, one vial was used to confirm $\mathrm{pH}$ and osmolarity.

\section{Statistical Analyses}

Outcome parameters post-treatment were compared with a pre-treatment baseline, using Wilcoxon rank-sum measures. Data are presented as median and interquartile range (IQR). A $P$ value of $<.05$ was considered significant. $P$ values considered to be significant were not modified in the setting of multiple comparisons.

\section{Results}

Ten subjects ( 7 male, 3 female, mean age 33.2 y) performed the glycine inhalation in study 1 . Eight subjects ( 6 male, 2 female, mean age 35.4 y) repeated the experiment 6 weeks later, inhaling standard normal saline instead of alkaline glycine. Two subjects had moved away during this time and were unable to complete this portion of the study. Ten subjects (4 male, 6 female, mean age $43.9 \mathrm{y})$ performed the second study. All of the subjects tolerated the inhaled glycine buffers. There were no ad- 


\section{Safety of an Alkalinizing Buffer Designed for Inhaled Medications in Humans}

Table. Median Changes in Physiologic Measurements From Baseline

\begin{tabular}{|c|c|c|c|c|c|c|}
\hline & \multicolumn{2}{|c|}{ Pre } & \multicolumn{2}{|c|}{ Post } & \multirow{2}{*}{$\% \Delta$} & \multirow{2}{*}{$P^{*}$} \\
\hline & Median & IQR & Median & IQR & & \\
\hline \multicolumn{7}{|c|}{ Initial study - saline group } \\
\hline $\mathrm{FEV}_{1}, \mathrm{~L}$ & 4.08 & $4.53-3.74$ & 4.24 & $4.56-3.68$ & 3.93 & .19 \\
\hline FVC, L & 5.06 & $5.58-4.88$ & 5.12 & $5.51-4.9$ & 1.19 & .81 \\
\hline $\mathrm{FEV}_{1} / \mathrm{FVC}, \%$ & 80.06 & $82.18-76.38$ & 81.69 & $81.90-78.62$ & 2.03 & .43 \\
\hline $\mathrm{FEF}_{25-75 \%}, \%$ & 3.59 & $4.48-3.36$ & 4.04 & $4.62-3.25$ & 12.69 & .85 \\
\hline $\mathrm{F}_{\mathrm{ENO}}, \mathrm{ppb}$ & 15 & $52-12$ & 15.5 & $43.5-10.5$ & 3.33 & .10 \\
\hline $\mathrm{EBC} \mathrm{pH}$ & 8.62 & $8.86-6.95$ & 8.59 & $8.83-8.37$ & NA & .95 \\
\hline \multicolumn{7}{|c|}{ Initial study - glycine group } \\
\hline $\mathrm{FEV}_{1}, \mathrm{~L}$ & 3.97 & $4.47-3.46$ & 3.99 & $4.25-3.52$ & 0.38 & .23 \\
\hline FVC, L & 4.96 & $5.51-4.61$ & 5.04 & $5.45-4.54$ & 1.61 & .62 \\
\hline $\mathrm{FEV}_{1} / \mathrm{FVC}, \%$ & 78.55 & $83.19-72.82$ & 79.44 & $81.68-72.08$ & 1.14 & .32 \\
\hline $\mathrm{FEF}_{25-75 \%}, \%$ & 3.72 & $4.89-2.83$ & 3.53 & $4.58-2.84$ & -5.11 & .04 \\
\hline $\mathrm{F}_{\mathrm{ENO}}, \mathrm{ppb}$ & 18.5 & $32-14$ & 14.5 & $32-10$ & -21.62 & .004 \\
\hline $\mathrm{EBC} \mathrm{pH}$ & 7.75 & $8.48-7.47$ & 7.85 & $8.40-7.56$ & NA & .85 \\
\hline \multicolumn{7}{|c|}{ Second study - glycine group } \\
\hline $\mathrm{FEV}_{1}, \mathrm{~L}$ & 2.76 & $3.48-2.58$ & 2.79 & $3.31-2.49$ & 1.09 & .36 \\
\hline FVC, L & 3.7 & $4.7-3.24$ & 3.47 & $4.41-3.17$ & -6.22 & .04 \\
\hline $\mathrm{FEV}_{1} / \mathrm{FVC}, \%$ & 78.6 & $83.1-74.0$ & 80.35 & $82.1-75.6$ & 2.23 & .50 \\
\hline $\mathrm{FEF}_{25-75 \%}, \%$ & 2.6 & $3.14-2.32$ & 2.84 & $3.25-2.17$ & 9.23 & .88 \\
\hline $\mathrm{F}_{\mathrm{ENO}}, \mathrm{ppb}$ & 23 & $43-16$ & 21.5 & $38-18$ & -6.52 & .38 \\
\hline $\mathrm{EBC} \mathrm{pH}$ & 7.71 & $8.27-7.17$ & 8.34 & $8.62-7.36$ & NA & .03 \\
\hline \multicolumn{7}{|c|}{$\begin{array}{l}\text { * The } P \text { values have not been adjusted for multiple comparisons. } \\
\mathrm{FEF}_{25-75 \%}=\text { forced expiratory flow during the middle half of the FVC maneuver } \\
\mathrm{F}_{\mathrm{ENO}}=\text { fraction of exhaled nitric oxide } \\
\mathrm{EBC}=\text { exhaled breath condensate }\end{array}$} \\
\hline
\end{tabular}

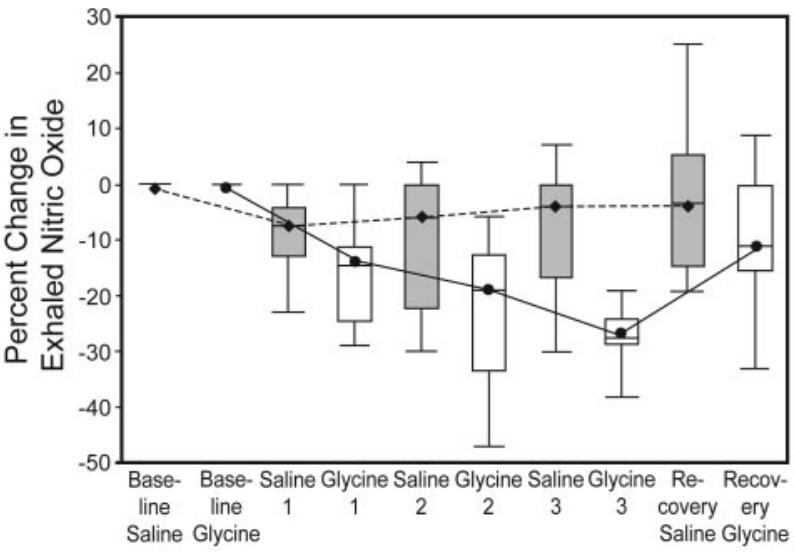

Fig. 2. Comparison of alkaline glycine to normal saline inhalation (study 1). Median percentage change in $F_{E N O}$ from baseline at 5 time points during the study: saline compared to glycine. Time 0 is baseline, followed by an $F_{E N O}$ measurement after each nebulization (alkaline glycine is represented by the white bars, normal saline is represented by the shaded bars). The final (recovery) measurement was taken 30 min later, prior to discharge from the clinical research unit. Each data bar shows the median (horizontal line inside the bar), upper and lower quartile (top and bottom of the bar), and minimum and maximum (whiskers). verse events of any kind. The changes in measurements for the studied groups from baseline throughout the studies are reported in the Table. The median change in $\mathrm{F}_{\mathrm{ENO}}$ from baseline after both glycine buffer and normal saline inhalation is reported in Figure 2. Vital signs were unchanged in all subjects throughout both studies. $\mathrm{F}_{\mathrm{ENO}}$ levels decreased by a median of $-21.62 \%(P=.004)$ after study 1 glycine inhalation, but there was no significant change in $\mathrm{F}_{\mathrm{ENO}}$ in study 2. The largest decreases in $\mathrm{F}_{\mathrm{ENO}}$ from baseline occurred in subjects with clinically elevated

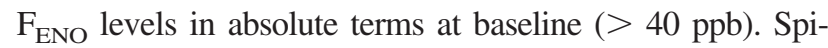
rometry levels remained within normal clinical ranges for both studies (see Table). There was no adverse effect on our primary safety outcome variable, $\mathrm{FEV}_{1}$. There was a slight decrease of $5.11 \%$ in $\mathrm{FEF}_{25-75 \%}$ after inhalation of study 1 glycine (IQR -12.579 to $0.292, P=.04$ ), which was not seen in study 2, and also of $6.22 \%$ in FVC after inhalation of study 2 glycine (IQR -2.16 to $-6.17, P=.04$, see Table), which was not seen in study 1 .

EBC $\mathrm{pH}$ increased by a median of $0.235 \mathrm{pH}$ units after inhalation of the study 2 alkaline glycine buffer (IQR $0.56-$ $0.03, P=.03$, Fig. 3), but had no statistically significant change after the study 1 treatments. 


\section{Safety of an Alkalinizing Buffer Designed for Inhaled Medications in Humans}

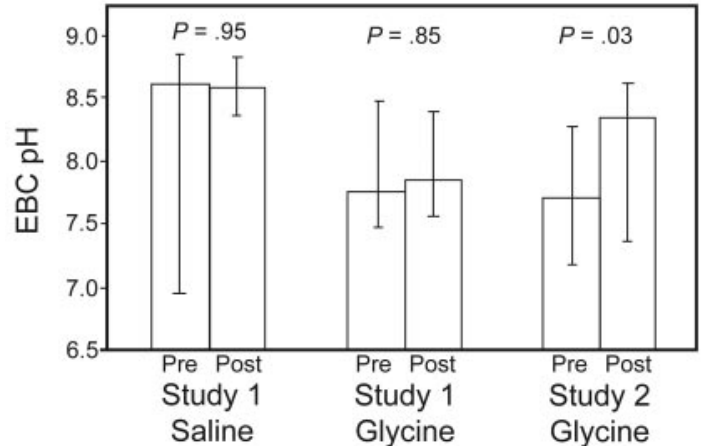

Fig. 3. Exhaled breath condensate $(E B C) \mathrm{pH}$ results from study 1 and study 2. Median EBC pH increased $0.235 \mathrm{pH}$ units $(P=.03)$ after inhalation of $120 \mathrm{mmol} / \mathrm{L}$ alkaline glycine, compared to no statistically significant change after low-dose alkaline glycine and normal saline.

\section{Discussion}

Our results provide additional data supporting the safety of inhaled alkaline glycine in healthy subjects and those with obstructive lung disease. It is possible to achieve increases in airway $\mathrm{pH}$ by means of inhaled alkaline glycine, and inhalation of alkaline glycine in healthy subjects and in subjects with obstructive lung disease causes no adverse effects on spirometric parameters. $\mathrm{EBC} \mathrm{pH}$ did increase, especially after inhalation of the higher-dose study 2 alkaline glycine, indicating the potential for efficacy as an airway alkalinization method. Subjects reported no subjective adverse effects after treatment with alkaline glycine. The EBC $\mathrm{pH}$ of one subject in the study 1 glycine group dropped below normal (7.18 after the study), and this subject had witnessed belching during the study (the sensitivity of $\mathrm{EBC} \mathrm{pH}$ to gastric acid reflux is well recognized ${ }^{22}$ ).

As shown in Figure 1, changes in $\mathrm{F}_{\mathrm{ENO}}$ after inhalation of the study 1 alkaline glycine were pronounced, compared to the study 1 saline group. Although it is possible that levels of $\mathrm{F}_{\mathrm{ENO}}$ can be lowered by spirometry, ${ }^{24}$ the statistically insignificant change in $\mathrm{F}_{\mathrm{ENO}}$ seen after saline inhalation effectively assures that the alkaline glycine was the primary cause of the $\mathrm{F}_{\mathrm{ENO}}$ decline. Although the EBC $\mathrm{pH}$ did not change significantly in the study 1 subjects, this does not preclude that alkalinization occurred. In many letters to editors, Effros et al contend that oral contamination of EBC by ammonia eliminates the ability of EBC to function as an indicator of ALF pH. ${ }^{25}$ While the weight of peer-reviewed published original research refutes that contention and supports the use of $\mathrm{EBC} \mathrm{pH}$ as one indicator of ALF $\mathrm{pH},{ }^{4,7,12,19,26}$ it seems certain to us that the sensitivity of $\mathrm{EBC} \mathrm{pH}$ for identifying airway acidity is indeed decreased when high levels of oral ammonia are present to neutralize exhaled airway acids. Thus, a low EBC pH is highly specific for airway acidification, but when oral am- monia release is high, $\mathrm{EBC} \mathrm{pH}$ is not as sensitive for airway acidification. This phenomenon could explain the lack of change in EBC pH in study 1. It is noted that in study 1 the baseline $\mathrm{F}_{\mathrm{ENO}}$ was higher, and $\mathrm{EBC} \mathrm{pH}$ and $\mathrm{FEV}_{1}$ were lower prior to the glycine inhalation, compared to the saline inhalation intervention given 6 weeks later. We speculate that this may result from the timing of the study in relation to allergy seasons, as the glycine intervention was performed in September (high allergy season in our region).

After inhaling alkaline glycine, the subjects in study 2 did not experience a statistically significant change in $\mathrm{F}_{\mathrm{ENO}}$, but, in contrast, did demonstrate a marked increase in EBC $\mathrm{pH}$. This finding supports the use of $\mathrm{EBC} \mathrm{pH}$ to detect acidic ALF pH, as several of the subjects in this study demonstrated acidic $\mathrm{EBC} \mathrm{pH}$ at baseline, consistent with known findings of low $\mathrm{EBC} \mathrm{pH}$ in patients with disease (median 7.71, IQR 7.17-8.27). These subjects had known obstructive airway disease, and many were receiving inhaled corticosteroid therapy, which is known to decrease nitric oxide synthase activity and net nitrogen oxide production. Corticosteroid therapy decreases $\mathrm{F}_{\mathrm{ENO}}$ at baseline, which likely explains the lack of an observed $\mathrm{F}_{\mathrm{ENO}}$ effect in study $2 .{ }^{27}$

Our studies had several limitations. The primary focus was on the safety of the inhaled alkaline glycine buffer; therefore, several pieces of data that would have strengthened the secondary end points were not measured. In study 1 the healthy controls were not screened for atopy prior to enrollment. This could explain the differences noted in baseline $\mathrm{F}_{\mathrm{ENO}}, \mathrm{EBC} \mathrm{pH}$, and spirometry levels in the same subjects between the glycine treatment and the saline treatment. In study 1, EBC pH was collected only at baseline and after the final nebulization of alkaline glycine buffer. This was done to minimize delays between the 3 nebulizations. We used change in $\mathrm{F}_{\mathrm{ENO}}$ after inhalation as a surrogate detector of airway $\mathrm{pH}$ changes at these time points, due to the rapidity of the testing $\left(\sim 11 \mathrm{~s}\right.$ for $\mathrm{F}_{\mathrm{ENO}}$, compared to $\sim 7 \mathrm{~min}$ for EBC collection). Since study 1 was the first human trial of this alkaline glycine buffer, it was necessary to deliver the compound through serial small doses, in order to evaluate its safety and detect potential adverse reactions prior to delivery of a larger dose. Given the full tolerability of the inhaled buffer, we increased the concentration of buffer so that the next phase would receive only one treatment.

In study 2 we wished to evaluate the effects of inhaled alkaline glycine on patients with obstructive lung function: specifically, on the physiologic issue of air-flow limitation. Although heterogeneous, all enrolled subjects had documented airway obstruction on the study day. We did not determine their degree of airway hyper-responsiveness nor responsiveness to $\beta$ agonists at the time of the study. We did not test the patients for atopy, immunologic dis- 


\section{Safety of an Alkalinizing Buffer Designed for Inhaled Medications in Humans}

ease, unrecognized environmental/occupational illness, or for any of the other numerous factors that could contribute to their obstruction. This current study was simply examining safety in patients with air-flow obstruction. In future studies we will be evaluating the efficacy of inhaled alkaline glycine buffer in patients with obstructed lower airways, and will use classification schemes and semi-specific disease names such as "asthma" and "COPD," to the extent that they are not misleading.

Also in study 2 we did not screen out subjects based on corticosteroid use. This may explain the lack of significant change in $\mathrm{F}_{\mathrm{ENO}}$ results, since corticosteroid therapy decreases $\mathrm{F}_{\mathrm{ENO}}$ at baseline. ${ }^{27} \mathrm{We}$ also did not serially measure $\mathrm{F}_{\mathrm{ENO}}$ and $\mathrm{EBC} \mathrm{pH}$ for time points after inhalation of the alkaline glycine buffer. In previous studies the greatest reduction in $\mathrm{F}_{\mathrm{ENO}}$ following inhalation of alkaline buffer occurred 15-60 min after inhalation. ${ }^{2,23}$ When using $\mathrm{F}_{\mathrm{ENO}}$ to detect changes in airway $\mathrm{pH}$, we recommend documenting and/or withholding corticosteroids prior to the study, and measuring $\mathrm{F}_{\mathrm{ENO}}$ at several time points in the hour following the study intervention.

Although the inhaled medication evaluated in this study was a solution (not a colloid), the formulation was different from the physiologic saline used by the manufacturer to determine the $2.1 \mu \mathrm{m}$ mass median aerodynamic diameter output of the nebulizer that was used in this study. It is possible that the mass median aerodynamic diameter output of the nebulizer of the study medication may be different from that of physiologic saline. This will be evaluated in future studies.

\section{Conclusions}

In conclusion, we report that the inhalation of isotonic alkaline glycine is safe in humans with or without obstructive airway disease, and that both $\mathrm{EBC} \mathrm{pH}$ and $\mathrm{F}_{\mathrm{ENO}}$ levels may be useful to indicate effective alkalinization of the airways. Future studies should evaluate the effects of alkaline therapy on subjects with known acute airway $\mathrm{pH}$ disturbances. Perhaps the most interesting near-term use of inhaled alkaline glycine is to improve the absorption across the airway epithelium of certain therapeutic agents that carry a charge at acidic but not alkaline $\mathrm{pH}$. Such therapeutics (including most $\beta$ agonists and anticholinergics) achieve better passive and active transfer through the airway epithelium - and therefore access to their smooth muscle target - when the ALF is alkaline. ${ }^{28}$ Because these medications are currently delivered at low $\mathrm{pH}$, and they are mostly used during acute respiratory illnesses, when the airways are most likely to be acidic, the possibility of improving drug delivery by means of alkalinization with glycine is currently undergoing clinical investigation.

\section{REFERENCES}

1. Ricciardolo FL, Gaston B, Hunt J. Acid stress in the pathology of asthma. J Allergy Clin Immunol 2004;113(4):610-619.

2. Gaston B, Kelly R, Urban P, Liu L, Henderson EM, Doctor A, et al. Buffering airway acid decreases exhaled nitric oxide in asthma. J Allergy Clin Immunol 2006;118(4):817-822.

3. Bunyan D, Smith A, Davidson W, Yu Y, Urban P, Naccara L, et al. Correlation of exhaled breath condensate $\mathrm{pH}$ with invasively measured airway pH in the cow (abstract). Eur Respir J 2005;26(49): 2407.

4. Horvath I, Hunt J, Barnes PJ. Exhaled breath condensate: methodological recommendations and unresolved questions. Eur Respir J 2005;26(3):523-548.

5. Walsh BK, Mackey DJ, Pajewski T, Yu Y, Gaston BM, Hunt JF. Exhaled-breath condensate $\mathrm{pH}$ can be safely and continuously monitored in mechanically ventilated patients. Respir Care 2006;51(10): 1125-1131.

6. Accordino R, Visentin A, Bordin A, Ferrazzoni S, Marian E, Rizzato $\mathrm{F}$, et al. Long-term repeatability of exhaled breath condensate $\mathrm{pH}$ in asthma. Respir Med 2008;102(3):377-381.

7. Hunt JF, Fang K, Malik R, Snyder A, Malhotra N, Platts-Mills TA, et al. Endogenous airway acidification. Implications for asthma pathophysiology. Am J Respir Crit Care Med 2000;161(3 Pt 1):694699 .

8. Brunetti L, Francavilla R, Tesse R, Strippoli A, Polimeno L, Loforese A, et al. Exhaled breath condensate pH measurement in children with asthma, allergic rhinitis and atopic dermatitis. Pediatr Allergy Immunol 2006;17(6):422-427.

9. Carpagnano GE, Barnes PJ, Francis J, Wilson N, Bush A, Kharitonov SA. Breath condensate $\mathrm{pH}$ in children with cystic fibrosis and asthma: a new noninvasive marker of airway inflammation? Chest 2004;125(6):2005-2010.

10. Carraro S, Folesani G, Corradi M, Zanconato S, Gaston B, Baraldi E. Acid-base equilibrium in exhaled breath condensate of allergic asthmatic children. Allergy 2005;60(4):476-481.

11. Gessner C, Hammerschmidt S, Kuhn H, Seyfarth HJ, Sack U, Engelmann L, et al. Exhaled breath condensate acidification in acute lung injury. Respir Med 2003;97(11):1188-1194.

12. Hunt J, Yu Y, Burns J, Gaston B, Ngamtrakulpanit L, Bunyan D, et al. Identification of acid reflux cough using serial assays of exhaled breath condensate $\mathrm{pH}$. Cough 2006;2:3.

13. Kostikas K, Papatheodorou G, Ganas K, Psathakis K, Panagou P, Loukides S. pH in expired breath condensate of patients with inflammatory airway diseases. Am J Respir Crit Care Med 2002; 165(10):1364-1370.

14. Nicolaou NC, Lowe LA, Murray CS, Woodcock A, Simpson A, Custovic A. Exhaled breath condensate $\mathrm{pH}$ and childhood asthma: unselected birth cohort study. Am J Respir Crit Care Med 2006; 174(3):254-259.

15. Niimi A, Nguyen LT, Usmani O, Mann B, Chung KF. Reduced pH and chloride levels in exhaled breath condensate of patients with chronic cough. Thorax 2004;59(7):608-612.

16. Paget-Brown AO, Ngamtrakulpanit L, Smith A, Bunyan D, Hom S, Nguyen A, et al. Normative data for $\mathrm{pH}$ of exhaled breath condensate. Chest 2006;129(2):426-430.

17. Rosias PP, Dompeling E, Dentener MA, Pennings HJ, Hendriks HJ, Van Iersel MP, et al. Childhood asthma: exhaled markers of airway inflammation, asthma control score, and lung function tests. Pediatr Pulmonol 2004;38(2):107-114.

18. Varnai VM, Ljubicic A, Prester L, Macan J. Exhaled breath condensate $\mathrm{pH}$ in adult Croatian population without respiratory disorders: how healthy a population should be to provide normative data? Arh Hig Rada Toksikol 2009;60(1):87-97. 


\section{Safety of an Alkalinizing Buffer Designed for Inhaled Medications in Humans}

19. Vaughan J, Ngamtrakulpanit L, Pajewski TN, Turner R, Nguyen TA, Smith A, et al. Exhaled breath condensate $\mathrm{pH}$ is a robust and reproducible assay of airway acidity. Eur Respir J 2003;22(6):889-894.

20. Greenwald R, Ferdinands JM, Teague WG. Ionic determinants of exhaled breath condensate $\mathrm{pH}$ before and after exercise in adolescent athletes. Pediatr Pulmonol 2009;44(8):768-777.

21. Rothe M, Becher G, Siemers R, Decker M. The pH-value of exhaled breath condensate - mainly influenced by exhaled volatile compounds. Eur Respir J 2005;26(49):2405.

22. Adachi H, Nguyen PH, Belardinelli R, Hunter D, Jung T, Wasserman K. Nitric oxide production during exercise in chronic heart failure. Am Heart J 1997;134(2 Pt 1):196-202.

23. Shin HW, Shelley DA, Henderson EM, Fitzpatrick A, Gaston B, George SC. Airway nitric oxide release is reduced after PBS inhalation in asthma. J Appl Physiol 2007;102(3):1028-1033.

24. Silkoff PE, Wakita S, Chatkin J, Ansarin K, Gutierrez C, Caramori
M, et al. Exhaled nitric oxide after beta2-agonist inhalation and spirometry in asthma. Am J Respir Crit Care Med 1999;159(3):940944.

25. Effros RM, Casaburi R, Porszasz J, Rehan V. Why conventional exhaled breath condensate $\mathrm{pH}$ studies cannot provide reliable estimates of airway acidification (letter). Chest 2011;140(4):1099.

26. Wells K, Vaughan J, Pajewski TN, Hom S, Ngamtrakulpanit L, Smith A, et al. Exhaled breath condensate $\mathrm{pH}$ assays are not influenced by oral ammonia. Thorax 2005;60(1):27-31.

27. Kharitonov SA, Yates DH, Barnes PJ. Inhaled glucocorticoids decrease nitric oxide in exhaled air of asthmatic patients. Am J Respir Crit Care Med 1996;153(1):454-457.

28. Horvath G, Schmid N, Fragoso MA, Schmid A, Conner GE, Salathe $\mathrm{M}$, et al. Epithelial organic cation transporters ensure $\mathrm{pH}$-dependent drug absorption in the airway. Am J Respir Cell Mol Biol 2007; 36(1):53-60. 